\title{
Dynamical Conditions for the Containment of HIV Infection by CD8+ T Cells - A Variable Structure Control Approach
}

\author{
Anet J. N. Anelone and Sarah K. Spurgeon
}

\begin{abstract}
In the study of the Human Immunodeficiency Virus (HIV) infection dynamics, the reproductive ratio is a wellknown tool which provides a steady-state condition to determine the outcome of the infection. This paper assesses the control of HIV by the immune response. Dynamical conditions for the containment of HIV infection by the HIV-specific CD8+ $T$ cell response are evaluated using a model of HIV dynamics in vivo in which HIV-infected cells are killed before they start producing new virion. The reachability paradigm from Variable Structure Control (VSC) theory is used to formulate a dynamical condition for immunity. Simulation results show that this reachability condition effectively monitors the immunological requirements to contain HIV. This work also suggests that the cytolytic killing mechanism of $\mathrm{CD8}+\mathrm{T}$ cells operates as a boundary layer control to contain HIV infection. Together, the findings indicate that in contrast to the reproductive ratio, the proposed VSC approach delivers a framework to assess the effects of nonlinear dynamics and uncertainty as well as providing a means to investigate immunotherapy strategies.
\end{abstract}

\section{INTRODUCTION}

Following infection with the Human Immunodeficiency Virus (HIV), CD4+ T cells are preferentially targeted and a state of Acquired Immune Deficiency Syndrome (AIDS) is subsequently observed in most patients. Unfortunately, the immune system is generally not able to enforce protective immunity [23], [21]. However, the HIV-specific CD8+ T cell response appears to play an important role in the containment of the infection. Early thoughts assumed that HIV-specific CD8+ T cells kill HIV-infected cells whilst they are producing new virion [22], [2], [14], [28] but recent findings suggest that HIV-specific CD8+ T cells kill HIV-infected cells via a cytolytic mechanism during the eclipse phase of the infection, before infected cells start producing new virion. Consequently, the HIV-specific CD8+ T cell response can be regarded as a feedback mechanism mounted by the immune system to oppose the dynamics of HIV pathogenesis. Thus, this yields a closed-loop system in which the HIV-specific $\mathrm{CD} 8+\mathrm{T}$ cell response acts as an inherent control. It is of current interest to understand how the HIV-specific CD8+ T cell response contains HIV infection [23], [12], [26]. Further, immunological requirements to eradicate HIV infection in vivo are not yet clearly defined. The reproductive ratio $R_{0}$ is a classical tool used in the study of virus dynamics to determine the progression of the infection [27], [24], [4]. By

A. J. N. Anelone is with the School of Engineering and Digital Arts, University of Kent, Canterbury, Kent CT2 7NT, United Kingdom a jna 5 ekent.ac.uk

S. K. Spurgeon is with the Department of Electronic and Electrical Engineering, University College London, Torrington Place, London, WC1E 7JE, United Kingdom s.spurgeon@ucl.ac.uk definition, the reproductive ratio is the number of new cells that a single infected cell produces in the absence of a target cell limitation [24], [27]. When $R_{0}<1$, the infection does not spread but when $R_{0} \geq 1$, the infection spreads. Therefore, the HIV-specific CD8+ T cell killing mechanism must enforce the reproductive ratio to be below unity in order to induce the containment of HIV infection. Since the expression for $R_{0}$ is an algebraic combination of some constant biological rates, this implies that the condition for the containment of HIV infection is time invariant and depends soley on the values of some biological rates rather than the population dynamics.

In contrast, recent findings suggest that immunological requirements to contain HIV infection change during the progression of infection [24], [28], [26]; the immunological requirements may be weaker before the peak of the virus load due to the small number of viral particles at the site of the infection [24]. Work in [11] suggests that the failure of the HIV-specific CD8+ T cell response to eradicate infection is explained by a low ratio between the number of effector HIV-specific CD8+ T cells and the population size of the infected cells (target) at an early stage of the infection and before the peak of the virus load. Hence, the framework of the reproductive ratio does not encompass the emerging notion that the condition for the containment of the infection depends on biological rates as well as the time evolution of $\mathrm{T}$ cells and virus dynamics.

The novelty and major contribution of this paper resides in the fact that the reachability paradigm from Variable Structure Control (VSC) theory provides a dynamical condition for the HIV-specific CD8+ $\mathrm{T}$ cell response to contain HIV infection and reach the infection-free steady-state. Furthermore, this condition encompasses the nonlinearities of the model and exhibits some robustness properties with respect to parameter and modelling uncertainty. A variety of control techniques have been considered to analyse HIV dynamics in vivo [9], [1], [25], [6]. Evaluating the condition for the containment of HIV infection by the HIV-specific CD8+ $\mathrm{T}$ cell response using a Variable Structure Control (VSC) approach is supported by the following observations: 1- the dynamics of the CD8+ $\mathrm{T}$ cell response has been shown to behave as a switched control system in [3]. 2- The double saturation function modelling cytolytic mechanism in [16] reveals that the structure of the killing action changes during the infection. 3- Using HIV clinical data, it has been shown in [4] that the reachability paradigm provides a suitable dynamical condition to predict the outcome of antiretroviral therapy. 
This paper is structured as follows: In section II, the chosen HIV dynamic model is presented. A dynamical condition for immunity using the VSC paradigm is derived in section III. The reproductive ratio is revisited and analyzed in section IV. Finally, in section V, simulations are conducted to support the analytical analysis and the notion that the dynamical condition for immunity from the reachability analysis is an appropriate tool to monitor the immunological requirements to contain infection.

\section{MODEL DESCRIPTION}

The model considered in this paper is as described in [2]. This system has been selected because its dynamical behaviour appropriately represents the acute phase of HIV infection [2], [10], [22]. This model assumes that HIVspecific CD8+ $\mathrm{T}$ cells control the infection by killing HIVinfected cells in the eclipse phase before they start producing new virion. A block diagram of this process is given in Fig. 1. The dynamical equations are as follows:

$$
\begin{aligned}
\frac{d T}{d t} & =\lambda-\delta_{T} T-\beta T V \\
\frac{d I}{d t} & =f \beta T V-\gamma I-\delta_{T} I+u_{C} \\
\frac{d P}{d t} & =\gamma I-\delta_{P} P \\
\frac{d V}{d t} & =p P-\delta_{V} V \\
\frac{d E}{d t} & =\frac{g_{\max } I}{h_{g}+I+E}-\delta_{E} E
\end{aligned}
$$

The definition of the states variables and parameters of the model (1) are listed in Table I. Details on the architecture of the model (1) can be found in [2], [10]. Here, the cytolytic

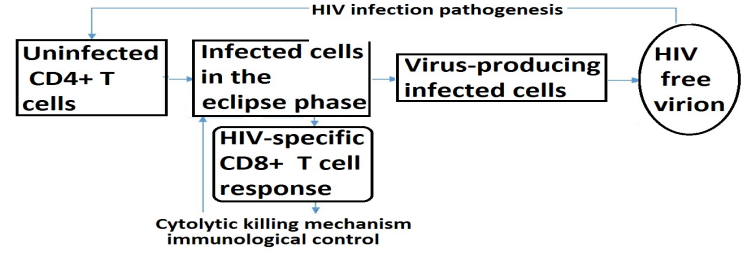

Fig. 1. Model (1) of HIV infection dynamics in which infected cells in the eclipse phase are killed by the cytolytic effect of CD8+ T cells

mechanism of HIV-specific CD8+ T cells, $u_{C}$, where $u_{C} \leq 0$, is treated as an intrinsic immunological control action. It is assumed that HIV-specific CD8+ T cells control/contain HIV infection in vivo via this cytolytic mechanism, i.e killing HIV infected cells in the eclipse phase [10], [2]. The non-cytolytic mechanism with range $0 \leq f \leq 1$ is not the focus and is set to $f=1$. However, it constitutes a candidate immunological means to contain the infection in vivo [14].

The infection free steady-state located at the trivial equilibrium of the model (1) is given as:

$$
T_{s 0}=\frac{\lambda}{\delta_{T}} ; \quad I_{s 0}=P_{s 0}=V_{s 0}=E_{s 0}=0
$$

Throughout the paper, the term condition for immunity is defined as the condition that the killing mechanism of the
TABLE I

LIST OF THE STATE VARIABLES AND PARAMETERS OF THE MODEL (1)

\begin{tabular}{|c|c|}
\hline Description & Symbol \\
\hline Uninfected CD4+ T cells & $T$ \\
\hline HIV-infected CD4+ T cells in the eclipse phase & $I$ \\
\hline HIV-infected CD4+ T cells producing new virion & $P$ \\
\hline HIV free virion & $V$ \\
\hline HIV-specific CD8+ T cells & $E$ \\
\hline Source rate of uninfected CD4+ T cells & $\lambda$ \\
\hline Death rate of uninfected CD4+ T cells & $\delta_{T}$ \\
\hline HIV infection rate & $\beta$ \\
\hline Non-cytolytic killing mechanism of CD8+ T cells & $f$ \\
\hline Rate of virus eclipse phase & $\gamma$ \\
\hline Cytolytic killing mechanism of CD8+ T cells & $u_{C}$ \\
\hline Death rate of virus-producing infected CD4+ T cells $P$ & $\delta_{P}$ \\
\hline Virus production rate & $\rho$ \\
\hline Virus clearance rate & $\delta_{V}$ \\
\hline Proliferation rate of HIV-specific CD8+ T cells & $g_{\max }$ \\
\hline Michaelis-Menten constant for proliferation & $h_{g}$ \\
\hline Death rate of HIV-specific CD8+ T cells & $\delta_{E}$ \\
\hline
\end{tabular}

CD8+ $\mathrm{T}$ cell response needs to satisfy in order to zero the state variable $I$, so that the population of HIV-infected CD4+ $\mathrm{T}$ cells in the eclipse phase reach and maintain the infectionfree steady-state. Thus, the control objective is to satisfy the condition for immunity.

\section{DYNAMICAL CONDITION FOR IMMUNITY USING THE REACHABILITY ANALYSIS}

As in [3], [4], the framework of sliding mode control is utilized to investigate the performance of the specific $\mathrm{T}$ cell response. Here, a sliding manifold representing the immunological objective of the HIV-specific CD8 $+\mathrm{T}$ cell response is defined and a reachability condition is formulated to ensure that immunity is achieved. The sliding surface is given as:

$$
s_{E}(t)=I
$$

In this sliding mode control approach, the HIV-specific CD8+ $\mathrm{T}$ cell response in the ideal case is thought to render the manifold $s_{E}=0$ attractive. In the sliding mode,

$$
s_{E}(t)=I=0 \quad \text { and } \quad \frac{d s_{E}}{d t}=0
$$

defines the dynamical behaviour of the system (1). In fact, the sliding manifold $s_{E}=0$ is selected so that ideal sliding motion yields a stable infection-free steady-state. It should be mentioned that the sign of $s_{E}(t)$ is non-negative at all times because it relates to the number of HIV-infected CD4+ T cells in the eclipse phase $I \geq 0$. The temporal derivative of the sliding surface is:

$$
\frac{d s_{E}}{d t}=\frac{d I}{d t}=f \beta T V-\left(\gamma+\delta_{T}\right) I+u_{C}
$$

Next, the concept of the equivalent control, introduced in [29], [13], [7] is considered. The equivalent control $u_{C e q}$, the effective control action required to maintain sliding motion on $s_{E}=0$, is retrieved from $\frac{d s_{E}}{d t}=0$ as follows:

$$
u_{C e q}=-f \beta T V+\gamma I+\delta_{T} I
$$


Hence, the equivalent control is meaningful and represents quantities that can be measured or estimated [18], [31], [27]. Here, the reachability condition, see [13], [29], [7], is given as:

$$
\begin{aligned}
s_{E} \frac{d s_{E}}{d t} & <0 \\
I\left(f \beta T V-\left(\gamma+\delta_{T}\right) I+u_{C}\right) & <0 \\
\left(f \beta T V-\left(\gamma+\delta_{T}\right) I+u_{C}\right) & <0
\end{aligned}
$$

since $0 \leq I$ by definition. This inequality highlights a timevarying threshold for the cytolytic killing of the HIV-specific CD8+ $\mathrm{T}$ cell response to zero the population of HIVinfected cells $I$ in the eclipse phase to ensure immunity. This dynamical condition for immunity is the main contribution of this paper. Importantly, this reachability analysis supports the notion that the condition for immunity changes during the course of the infection [24], [23], [11].

The expression (7) provides a means to clarify the effects of uncertainty in the values of biological rates and population dynamic of the system (1) on the containment of the infection. In fact, the work in [11], [10], [20] on modelling HIV dynamics in vivo pointed out that some of the constant parameters in (1) represent biological rates which vary according to time or to some population dynamics during the course of the infection. For instance, the infection rate $\beta$ is influenced by the antibody response to HIV [30]. This dynamical condition also infers that the negative feedback provided by the HIV-specific CD8+ T cell response should overcome the positive feedback of HIV pathogenesis encompassed by the product $\beta T V$ because the term $\beta T V$ is a dominant component which sustains HIV pathogenesis. In [11], [10], the mathematical representation of the HIVspecific CD8+ $\mathrm{T}$ cell response has been shown to influence the immunological requirement to contain the infection. This reachability analysis proves that the dynamical condition for immunity is invariant to the explicit mathematical formulation of the cytolytic or non-cytolytic killing mechanism of the HIV-specific CD8+ $\mathrm{T}$ cell response. This control analysis supports the notion that both the cytolytic and noncytolytic killing mechanism of HIV-specific CD8+ T cells are important to enforce immunity [20], [15], [14]. A number of mathematical approaches based on different biological assumptions have been proposed to model the HIV-specific CD8+ T cell response [20], [10], [11]. Functional responses inspired from ecology or Michaelis-Menten functions from enzyme-substrate kinetics are often used [10], [2], [16]. Typically, functions modelling the cytolytic killing mechanism of $\mathrm{CD} 8+\mathrm{T}$ cells have the following form:

$$
u_{C}=-\mathscr{K} I
$$

where $\mathrm{CD} 8+\mathrm{T}$ cells kill infected cells at a rate $\mathscr{K}$ which encompasses the cytolytic killing process [20], [27]. This immunological control action is effectively a negative state feedback which opposes the infection dynamics in vivo. A reasonable assumption is to consider that the cytolytic killing rate of HIV-specific $\mathrm{T}$ cells changes during the time course of the infection [20], [11]. Recently, a double-saturation
(DS) function which is a general functional response of the cytolytic killing mechanism of CD8+ $\mathrm{T}$ cells has been formulated in [16] as follows:

$$
u_{C 2}=-\mathscr{K}_{2}(t) I=-\frac{k^{\prime} E I}{1+E / h_{E}+I / h_{I}}
$$

where $h_{E}$ and $h_{I}$ are the saturation constant of the population of HIV-specific CD8+ T cells $E$ and the population of infected cells (target) in the eclipse phase $I$ respectively. The parameter $k^{\prime}$ is a mass action killing rate. The specific case of a monogamous killing process derived from (9) is considered to build on the findings in [2] as well as to demonstrate the benefits of the dynamical condition for immunity over the reproductive ratio. This monogamous killing process describes a cytolytic mechanism where a single effector CD8+ T cell kills target cells. Mathematically, this monogamous killing process is expressed as:

$$
u_{C 3}=-\frac{k_{\max } E I}{h_{k}+E+I}
$$

with $k^{\prime}=\frac{k_{\max }}{h_{k}}$ and $h_{k}=h_{E}=h_{I}$. The maximum killing rate is $k_{\max }$ and the parameter $h_{k}$ which is effectively a Michaelis-Menten constant defines the immune responsiveness [2], [16]. Tailoring the reachability condition to (10), the candidate immunological control action produces:

$$
f \beta T V-\left(\gamma+\delta_{T}\right) I-\frac{k_{\max } E I}{h_{k}+E+I}<0
$$

In [5], [13], it has been seen that when a continuous approximation of the discontinuous $\operatorname{sign}()$ function is implemented, ideal sliding motion can longer be achieved. Instead, the applied continuous control action keeps the states of the system within a vicinity of the sliding manifold. Consequently, using the results of [7], [5], [13] on smoothed variable structure control systems, it is argued that the control action of the cytolytic mechanism of the HIV-specific response modelled with the DS function (9) rather than zeroing the population of infected cells, confines its population size to a steady-state level determined by the magnitude of the cytolytic killing action. This suggests that the cytolytic mechanism of the $\mathrm{CD} 8+\mathrm{T}$ cell response operates as a boundary layer control mechanism in which the population size of target cells and virus-specific CD8+ T cells and their corresponding saturation constant determine the ability of the cytolytic killing action of virus-specific CD8+ T cells to "control" the infection and to achieve immunity [2], [11], [16], [17].

\section{STEADY-STATE CONDITION FOR IMMUNITY PROVIDED BY THE REPRODUCTIVE RATIO}

The concept of the reproductive ratio is reviewed from a control engineering perspective. In the study of virus dynamics, the reproductive ratio represents the number of newly infected cells produced by one infected cell during its lifetime, assuming there is no limitation of target cells [24]. 
The expression of the reproductive ratio derived in [2] using the infection-free steady-sate of model (1) is as follows:

$$
R_{0}=\frac{\lambda}{\delta_{T}} \frac{\gamma}{\gamma+\left(\delta_{T}+\mathscr{K}\right)} \frac{1}{\delta_{P}} \frac{p}{\delta_{V}} f \beta
$$

where the first factor is the population size of $T$ at the infection-free steady-state see (2). The second factor is the probability that an infected cell becomes a virus-producing cell before it dies. The third factor is the lifetime of a virusproducing cell. The fourth factor is the number of virus particles produced per infected cell, and the last parameter represents the infection rate for a single virus particle in presence of the non-cytolytic effects of HIV-specific CD8+ $\mathrm{T}$ cells.

The reproductive ratio plays a role in assessing the stability of the system (1) linearised at the infection-free steady-state (2). When $R_{0}<1$, the characteristic polynomial has negative poles and it is deduced that the infection-free steady-state is stable, whilst $R_{0}>1$ produces a positive pole. As a result, the infection-free steady state is unstable. This is why the infection is eradicated when $R_{0}<1$ and spreads when $R_{0} \geq 1$. This feature of $R_{0}$ [2], [22] has been used as evidence that the condition for the stability of the infection-free steadystate, i.e the outcome of the infection using the reproductive ratio (12), is a steady-state argument. Indeed, the poles of the system (1) change during the time evolution of the state variables i.e the time course of the infection. As a result, the value of $R_{0}$ is inappropriate to determine the outcome of the infection when the system is not at steady-state. Hence, the reproductive ratio has limitations as a means to monitor dynamically the containment of the infection by the HIVspecific CD8+ $\mathrm{T}$ cell response.

Using the framework of the reproductive ratio, the cytolytic killing rate of HIV-specific CD8+ T cells should force and maintain the reproductive ratio (12) below unity to eradicate the infection [24], [27], [2]. Mathematically, for $R_{0}<1$,

$$
\mathscr{K}>\frac{f \beta \lambda \gamma p-\delta_{T} \gamma \delta_{P} \delta_{V}}{\delta_{V} \delta_{T} \delta_{P}}-\delta_{T}
$$

Since the biological rates present in the right hand side are all constant, the framework of the reproductive ratio suggests that the condition for the cytolytic killing mechanism to eradicate the infection in vivo is time invariant. However, this is not in line with recent findings which indicate that the requirements to contain HIV infection in vivo change during the progression of the infection [24], [8], [23].

To investigate the predictions suggested by the reproductive ratio during the time evolution of the infection, the reproductive ratio is written as a function of the candidate control action (10) as follows:

$$
R_{0}=\frac{\lambda \gamma p f \beta}{\delta_{T} \delta_{P} \delta_{V}\left(\gamma+\left(\delta_{T}+\frac{k_{\max } E}{h_{k}+I+E}\right)\right)}
$$

This expression has been proposed in [2] and the value of $R_{0}$ changes over time due to changes in the killing rate of $u_{C 3}$ during the course of the infection.

In the following, simulations are conducted to evaluate the
TABLE II

VALUES OF BIOLOGICAL RATES

\begin{tabular}{|c|c|}
\hline Parameter & Value \\
\hline$\lambda$ & $210^{5}\left(\frac{\text { cells }}{\text { day }}\right)$ \\
\hline$\delta_{T}$ & $0.1\left(\right.$ day $\left.^{-1}\right)$ \\
\hline$\beta$ & $310^{-8}\left(\right.$ day $\left.^{-1}\right)$ \\
\hline$f$ & $1\left(\right.$ day $\left.^{-1}\right)$ \\
\hline$\gamma$ & $1\left(\right.$ day $\left.^{-1}\right)$ \\
\hline$\delta_{I}$ & $0.1\left(\right.$ day $\left.^{-1}\right)$ \\
\hline$\delta_{P}$ & $1\left(\right.$ day $\left.^{-1}\right)$ \\
\hline$\rho$ & $2300\left(\right.$ day $\left.^{-1}\right)$ \\
\hline$\delta_{V}$ & $23\left(\right.$ day $\left.^{-1}\right)$ \\
\hline$g$ & $1.5\left(\right.$ day $\left.^{-1}\right)$ \\
\hline$h_{g}$ & 0 cells $^{-1}$ \\
\hline$\delta_{E}$ & $0.5\left(\right.$ day $\left.^{-1}\right)$ \\
\hline \multicolumn{2}{|r}{}
\end{tabular}

predictions of the reachability analysis and the reproductive ratio during the time evolution of HIV infection.

\section{Simulation Results}

HIV infection dynamics are simulated to show that the dynamical condition for immunity (7) provided by the reachability analysis appropriately tracks the immunological requirements for immunity during the course of the infection and is superior to the steady-state condition (13) formulated using the reproductive ratio (14). In the simulations, the initial condition depicts a scenario where the population of uninfected CD4+ T cells is at steady-state $T(0)=\frac{\lambda}{d_{1}}$, the infection is assumed to be induced by a single virion $I(0)=P(0)=0 ; V(0)=1$ and precursor HIV-specific CD8+ $\mathrm{T}$ cells $E(0)=1$. The parameter values shown in Table II are taken from [2].

The purpose of Fig. 2 is to display the time evolution of the biological population dynamics of the closed-loop system (1) with the monogamous cytolytic killing action (10) is the control. The transient dynamics and the steady-state of the dynamic of free-virion is reduced by the cytolytic killing of HIV-infected cells $I$ by the HIV-specific CD8+ T cell response. Fig. 3 shows that the value of the reproductive ratio during the time course of the infection. As per the numerical analysis in [2], $R_{0}$ approaches unity at steadystate when the control action (10) is applied. Importantly, the value of the reproductive ratio during the transient dynamics of the system is misleading in assessing the outcome of the infection because the value of $R_{0}$ fluctuates above and below unity during the transient phase. $R_{0}<1$ wrongly suggests the eradication of the infection from day 0 to day 10 and from day 18 to day 22, see Fig. 3. This is due to the fact that the poles of the system (1) vary during the infection before the population dynamics reach a steady-state. This shows the limitation of the reproductive ratio to reflect appropriately the condition for immunity when the system is not at steadystate.

The time evolution of the reachability condition displayed in Fig. 4 reveals that the condition for immunity is not met at the beginning of the infection in contrast with the prediction of the reproductive ratio. Although, the considered control 


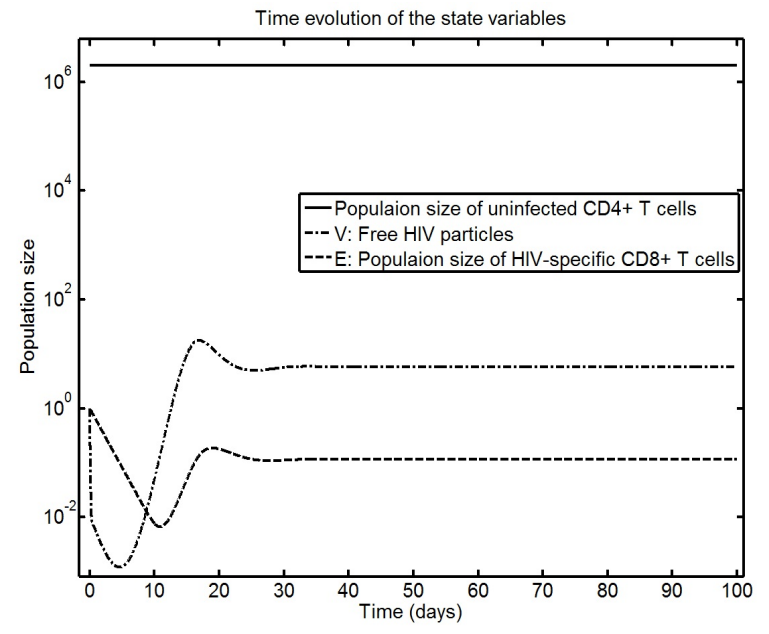

Fig. 2. Closed-loop dynamics (1). Simulation of HIV infection with a potent HIV-specific CD8+ response with $u_{C 1} . T(0)=2.10^{6} ; I(0)=P(0)=0$; $V(0)=E(0)=1 . f=1 ; h_{k}=1$

action $u_{C 3}$ reduces significantly the virus load, it does satisfy the dynamical condition for immunity (11) and the manifold of interest $s_{E}=0$ is not reached (see Fig. 5). Nevertheless, it should be noted that the candidate monogamous cytolytic killing action $u_{C 3}$ contains the infection to a low level steadystate close to the infection-free steady-state by keeping the sliding surface close to the desired manifold after a transient period (see Fig. 5). This is typical behaviour of a boundary layer control mechanism [5], [13]. The virus load is below the limit of detection which is 50 RNA copies per $\mathrm{ml}$ from [2], [19] and the population of uninfected CD4+ $\mathrm{T}$ cells is near its infection-free equilibrium. Therefore, from a clinical perspective, this candidate cytolytic killing mechanism produces a desirable outcome. Consequently, the condition for immunity can be relaxed to force the infection dynamics to remain within a boundary region associated with the virus limit of detection and other clinical requirements. Mathematically, this means that the condition for immunity is reduced to $s_{E}<\delta$ where $\delta$ is an upper bound associated with desirable clinical outcome. Thus, the cytolytic control functions which maintain the sliding surface in the vicinity of the sliding manifold $s_{E}=0$ can be classified as elite HIVspecific CD8+ response when the control action forces the virus load to remain below the limit of detection [23], [2], [14], [8]. In [2], it has been shown using numerical analysis that the value of $h_{k}$ affects the steady-state population size of infected cells $I$ and the steady-state level of the virus load. Using the sliding mode framework, changes in the saturation constant $h_{k}$ influence the magnitude of the control effort (killing action) $u_{C 3}$ as well as distance between the manifold of interest $s_{E}=0$ and the value of $s_{E}$ generated by $u_{C 3}$ with a particular value of $h_{k}$. This reinforces the notion that the saturation constant plays an important role in achieving immunity [16], [2]. Thus, failure of the reachability condition explains why this cytolytic killing mechanism fails to zero the population dynamic of infected cells $I$ and to eradicate the infection. Importantly, these findings cannot be substantiated

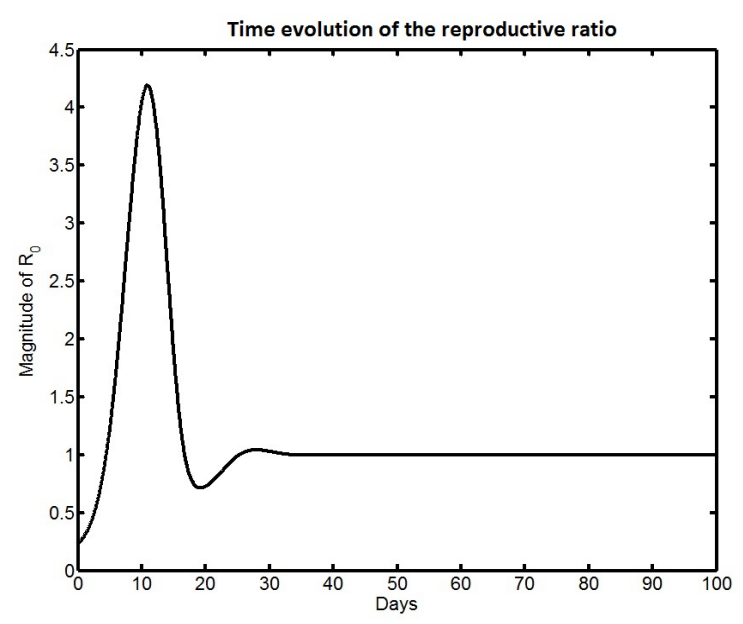

Fig. 3. Time evolution of the reproductive ratio (14) using $u_{C 3}$ (10) with $h_{l}=1$.

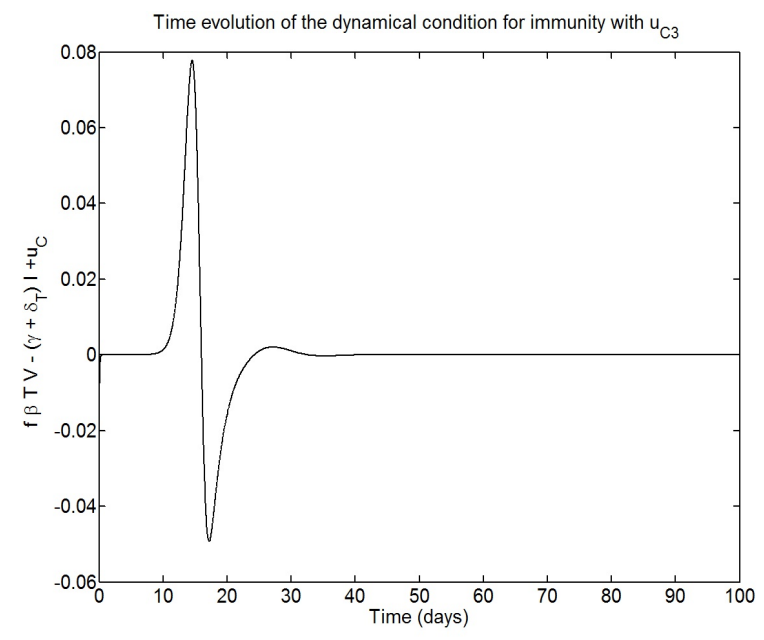

Fig. 4. Time evolution of the dynamical condition for immunity (11) with the cytolytic killing mechanism of the HIV-specific CD8+ T cell response $u_{C 3}(10)$

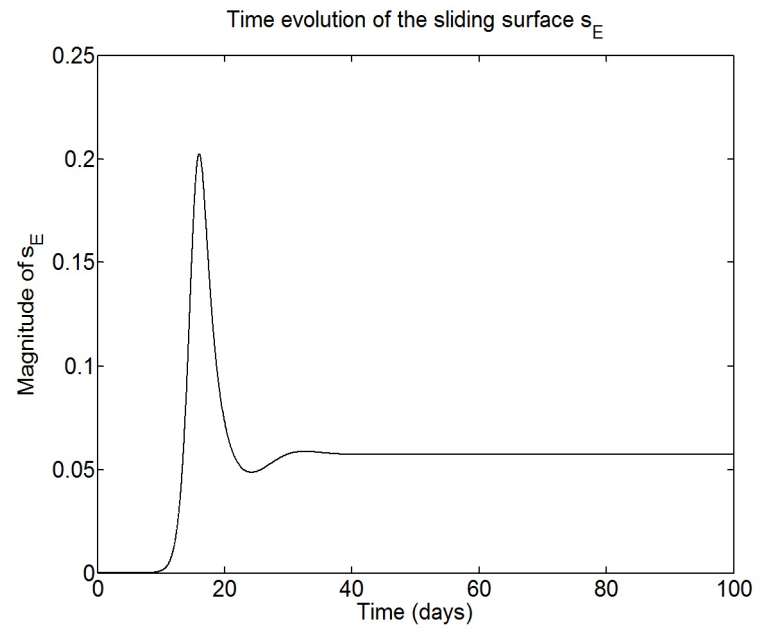

Fig. 5. Time evolution of the sliding surface (3) with $u_{C 3}$ (10). 
using the reproductive ratio because $R_{0}$ suggests just that the infection will decay but does not articulate the decay of the infection in finite time or decay to zero.

\section{CONCLUSION}

The reachability paradigm from VSC has been used to formulate a time-varying condition for the HIV-specific $\mathrm{CD} 8+\mathrm{T}$ cell response to contain HIV infection in vivo by cytolytic killing of the population of HIV-infected cells in the eclipse phase. A manifold associated with the infectionfree steady-state has been identified and the reachability condition for the system to exhibit a sliding mode is used to provide a dynamical condition for the cytolytic mechanism to contain the infection. Unlike the reproductive ratio, this dynamical condition for immunity has been demonstrated to monitor the immunological requirements for immunity during the time evolution of the infection appropriately. Furthermore, the cytolytic mechanism has been shown to operate as an inherent boundary layer control mechanism which confines the virus dynamics in a region of the statespace. Together the results support the notion that the condition for the eradication of HIV infection changes during the course of the infection and the ability of the HIV-specific cytolytic mechanism to induce immunity is influenced by the population size of infected cells and effector CD8+ cells and their corresponding saturation constant. Importantly. this dynamical condition may assist investigations to combine antiretroviral drugs and immunotherapy to achieve functional cure of HIV infection.

\section{REFERENCES}

[1] F. A. Alazabi and M. A. Zohdy. Nonlinear uncertain HIV-1 model controller by using control Lyapunov function. International J. of Modern Nonlinear Theory and App., 1:33 - 39, 2012.

[2] C. L. Althaus and $R_{i}$ J. De Boer. Implications of CTL-mediated killing of HIV-infected cells during the non-productive stage of infection. PLOS ONE, 6(2): e16468, 022011.

[3] A. J. N. Anelone and S. K. Spurgeon. Modelling and simulation of the dynamics of the antigen-specific $\mathrm{T}$ cell response using variable structure control theory. PLOS ONE, 11(11): e0166163, 112016.

[4] A. J. N. Anelone and S. K. Spurgeon. Prediction of the containment of HIV infection by antiretroviral therapy a variable structure control approach. IET Systems Biology, 11 (1):44 53, 02 2017. .

[5] J. A. Burton and A. S. I. Zinober. Continuous approximation of variable structure control. Int. J. Systems Sci., 17:876-885, 1986.

[6] H. Chang and A. Astolfi. Immune response enhancement via controlled drug scheduling. In Decision and Control, 2007 46th IEEE Conference on, pages 3919-3924, 2007.

[7] M. Chen, Y. Hwang, and M. Tomizuka. A state-dependent boundary layer design for sliding mode control. IEEE Transactions on Automatic Control, 47(10):1677-1681, Oct 2002.

[8] J. M. Conway and A. S. Perelson. Post-treatment control of HIV infection. Proceedings of the National Academy of Sciences, 112(17):54675472, 2015.

[9] B.A. Costa and J.M. Lemos. Nonlinear feedback control of a HIV-1 infection model. In Control Automation (MED), 2011 19th Mediterranean Conference on, pages 79-84, 062011.

[10] R. J. De Boer. Which of our modeling predictions are robust? PLOS Computat Biol, 8(7):e1002593, 072012.

[11] R. J. De Boer. Understanding the failure of CD8+ T-cell vaccination against Simian/Human Immunodeficiency Virus. Journal of Virology, 81(6):2838-2848, 2007.

[12] S. G. Deeks and B. D. Walker. Human Immunodeficiency Virus controllers: Mechanisms of durable virus control in the absence of antiretroviral therapy. Immunity, 27(3):406 - 416, 2007.
[13] C. Edwards and S. K. Spurgeon. Sliding mode control: theory and applications. CRC Press, 1998.

[14] M. Elemans, N. S. Al Basatena, and B. Asquith. The efficiency of the human CD8+ T cell response: How should we quantify it, what determines it, and does it matter? PLOS Comput Biol, 8(2):e1002381, 022012.

[15] M. Elemans, N. S. Al Basatena, N R. Klatt, C. Gkekas, G. Silvestri, and B. Asquith. Why don't CD8+ T cells reduce the lifespan of sivinfected cells in vivo? PLOS Comput Biol, 7(9):e1002200, 092011.

[16] S. Gadhamsetty, A.F.M. Mare, J.B. Beltman, and R.J. de Boer A general functional response of cytotoxic T lymphocyte-mediated killing of target cells. Biophysical Journal, 106(8):1780 - 1791, 2014.

[17] V.V. Ganusov, D.L. Barber, and R.J. De Boer. Killing of targets by $\mathrm{CD} 8+\mathrm{T}$ cells in the mouse spleen follows the law of mass action. PLOS ONE, 6(1):e15959, 012011.

[18] A M Jeffrey and X Xia. Identifiability of HIV/AIDS model In: W.Y. Tan, H. Wu (Eds.), Deterministic and Stochastic Models of AIDS Epidemics and HIV Infections with Intervention. World Scientific, Singapore, 2005.

[19] M. Mhawej, C. Brunet-Franois, R. Fonteneau, D. Ernst, V. Ferr, G.B. Stan, F. Raffi, and C. H. Moog. Apoptosis characterizes immunological failure of HIV infected patients. Control Engineering Practice, 17(7):798 - 804, 2009.

[20] V. Müller, A. F. M. Marée, and R. J. De Boer. Small variations in multiple parameters account for wide variations in HIV -1 set-points: a novel modelling approach. Proceedings of the Royal Society of London B: Biological Sciences, 268(1464):235-242, 2001.

[21] K. Murphy, P. Travers, M. Walport, and C. Janeway. Janeway's immunobiology. Garland Science, New York, 8th edition, 2012.

[22] A. S. Perelson and P. W. Nelson. Mathematical analysis of HIV-1 dynamics in vivo. SIAM Review, 41:3-44, 1998.

[23] A. S. Perelson and R. Ribeiro. Modeling the within-host dynamics of HIV infection. BMC Biology, 11(1):96, 2013.

[24] R. M. Ribeiro, L. Qin, L. L. Chavez, D. Li, S. G. Self, and A. S. Perelson. Estimation of the initial viral growth rate and basic reproductive number during acute HIV-1 infection. Journal of Virology, 84(12):6096-6102, 2010.

[25] S. Sam Ge, Z. Tian, and T.H. Lee. Nonlinear control of a dynamic model of HIV-1. Biomedical Engineering, IEEE Transactions on, 52(3):353-361, 032005.

[26] N.M.G. Smith, P. Mlcochova, S.A. Watters, M.M.I. Aasa-Chapman, N. Rabin, S. Moore, S.G. Edwards, J.A. Garson, P.R. Grant, R.B. Ferns, A. Kashuba, N.P. Mayor, J. Schellekens, S.G.E. Marsh, A.J. McMichael, A.S. Perelson, D. Pillay, N. Goonetilleke, and R.K. Gupta. Proof-of-principle for immune control of global HIV-1 reactivation in vivo. Clinical Infectious Diseases, 2015.

[27] M. A. Stafford, L. Corey, Y. Cao, E. S. Daar, D. D. Ho, and A. S. Perelson. Modeling plasma virus concentration during primary HIV infection. Journal of Theoretical Biology, 203(3):285 - 301, 2000.

[28] G. B Stan, F. Belmudes, R. Fonteneau, F. Zeggwagh, M.-A. Lefebvre, C. Michelet, and D. Ernst. Modelling the influence of activationinduced apoptosis of CD4+ and CD8+ T-cells on the immune system response of a HIV-infected patient. Systems Biology, IET, 2(2):94-102, 032008.

[29] V.I. Utkin. Variable structure system with sliding mode. IEEE Trans. on Control Systems Technology, 10(6):780-792, 1977.

[30] N. K. Vaidya, R. M. Ribeiro, C. J. Miller, and A. S. Perelson. Viral dynamics during primary Simian Immunodeficiency Virus infection: Effect of time-dependent virus infectivity. Journal of Virology, 84(9):4302-4310, 2010.

[31] H. Wu, H. Zhu, H. Miao, and A. S Perelson. Parameter identifiability and estimation of HIV/AIDS dynamic models. Bulletin of mathematical biology, 70(3):785-799, 2008. 